\title{
Uniqueness of quantum states compatible with given measurement results
}

\author{
Jianxin Chen, ${ }^{1,2}$ Hillary Dawkins, ${ }^{1}$ Zhengfeng Ji, ${ }^{2,3}$ Nathaniel Johnston, ${ }^{1,2}$ David Kribs,,${ }^{1,2}$ Frederic Shultz, ${ }^{4}$ and Bei Zeng ${ }^{1,2}$ \\ ${ }^{1}$ Department of Mathematics \& Statistics, University of Guelph, Guelph, Ontario, Canada \\ ${ }^{2}$ Institute for Quantum Computing, University of Waterloo, Waterloo, Ontario, Canada \\ ${ }^{3}$ State Key Laboratory of Computer Science, Institute of Software, Chinese Academy of Sciences, Beijing, China \\ ${ }^{4}$ Mathematics Department, Wellesley College, Wellesley, Massachusetts 02481, USA \\ (Received 2 April 2013; published 11 July 2013)
}

\begin{abstract}
We discuss the uniqueness of quantum states compatible with given measurement results for a set of observables. For a given pure state, we consider two different types of uniqueness: (1) no other pure state is compatible with the same measurement results and (2) no other state, pure or mixed, is compatible with the same measurement results. For case (1), it was known that for a $d$-dimensional Hilbert space, there exists a set of $4 d-5$ observables that uniquely determines any pure state. We show that for case (2), $5 d-7$ observables suffice to uniquely determine any pure state. Thus, there is a gap between the results for (1) and (2), and we give some examples to illustrate this. Unique determination of a pure state by its reduced density matrices (RDMs), a special case of determination by observables, is also discussed. We improve the best-known bound on local dimensions in which almost all pure states are uniquely determined by their RDMs for case (2). We further discuss circumstances where (1) can imply (2). We use convexity of the numerical range of operators to show that when only two observables are measured, (1) always implies (2). More generally, if there is a compact group of symmetries of the state space which has the span of the observables measured as the set of fixed points, then (1) implies (2). We analyze the possible dimensions for the span of such observables. Our results extend naturally to the case of low-rank quantum states.
\end{abstract}

DOI: 10.1103/PhysRevA.88.012109

PACS number(s): 03.65.Ud, 03.67.Mn, 89.70.Cf

\section{INTRODUCTION}

In a $d$-dimensional Hilbert space $\mathcal{H}_{d}$, the description of any quantum state $\rho$ generated by a source can be obtained by quantum tomography. For any density matrix $\rho$, which is Hermitian and has trace $1, d^{2}-1$ independent measurements are sufficient and necessary to uniquely specify $\rho$. When $\rho=|\psi\rangle\langle\psi|$ is a pure state, one may not need as many measurements to uniquely determine $|\psi\rangle$. As we will see later, however, exactly what is meant by "uniquely" in this context needs to be specified.

Consider a set of $m$ linearly independent observables

$$
\mathbf{A}=\left(A_{1}, A_{2}, \ldots, A_{m}\right),
$$

where each $A_{i}$ is Hermitian. Measurements on state $\rho$ with respect to these observables give the following average values:

$$
\mathbf{A}(\rho):=\left(\operatorname{tr} \rho A_{1}, \operatorname{tr} \rho A_{2}, \ldots, \operatorname{tr} \rho A_{m}\right) \in \mathbb{R}^{m} .
$$

We denote the set of these $\mathbf{A}(\rho)$ for all states $\rho$ as

$$
C_{m}(\mathbf{A}):=\left\{\mathbf{A}(\rho): \rho \text { acts on } \mathcal{H}_{d}\right\} .
$$

For a pure state $|\psi\rangle$, these values are given by

$$
\mathbf{A}(|\psi\rangle):=\left(\left\langle\psi\left|A_{1}\right| \psi\right\rangle,\left\langle\psi\left|A_{2}\right| \psi\right\rangle, \ldots,\left\langle\psi\left|A_{m}\right| \psi\right\rangle\right),
$$

and we denote the set of these values for all pure states $|\psi\rangle$ as the joint numerical range

$$
W_{m}(\mathbf{A}):=\left\{\mathbf{A}(|\psi\rangle):|\psi\rangle \in \mathcal{H}_{d}\right\} .
$$

In this work, we consider two different kinds of "unique determinedness" for $|\psi\rangle$ :

(1) We say $|\psi\rangle$ is uniquely determined among pure states (UDP) by measuring $\mathbf{A}$ if there does not exist any other pure state which has the same measurement results as those of $|\psi\rangle$ when measuring $\mathbf{A}$.
(2) We say $|\psi\rangle$ is uniquely determined among all states (UDA) by measuring $\mathbf{A}$ if there does not exist any other state, pure or mixed, which has the same measurement results as those of $|\psi\rangle$ when measuring $\mathbf{A}$.

It is known that there exists a family of $4 d-5$ observables such that any pure state is UDP, in contrast to the $d^{2}-1$ observables in the general case of quantum tomography [1]. The physical meaning for this case is clear: it is useful for the purpose of quantum tomography to have the prior knowledge that the state to be reconstructed is pure or nearly pure. Many other techniques for pure state tomography have been developed, and experiments have been performed to demonstrate the reduction of the number of measurements needed [2-8].

When the state is UDP, to make the tomography meaningful, one needs to make sure that the state is indeed pure. This is not in general practical, but one can readily generalize the abovementioned UDP results to low-rank states, where the physical constraints (e.g., low temperature, locality of interaction) may ensure that the actual physical state (which ideally supposed to be pure) is indeed low rank. If the state is UDA, however, in terms of tomography one does not need to bother with these physical assumptions because in the event there is only a unique state compatible with the measurement results, which turns out to be pure (or low rank).

There is also another clear physical meaning for the states that are UDA by measuring A. Consider a Hamiltonian of the form

$$
H_{\mathrm{A}}=\sum_{i=1}^{m} \alpha_{i} A_{i} .
$$

Then, any unique ground state $|\psi\rangle$ of $H_{\mathbf{A}}$ is UDA by measuring A. This is easy to verify: if there is any other state $\rho$ that gives the same measurement results, then $\rho$ has the same energy 
as that of $|\psi\rangle$, which is the ground-state energy. Therefore, any pure state in the range of $\rho$ must also be a ground state, which contradicts the fact that $|\psi\rangle$ is the unique ground state. In other words, UDA is a necessary condition for $|\psi\rangle$ to be a unique ground state of $H_{\mathbf{A}}$. It is in general not sufficient, but the exceptions are likely rare $[9,10]$.

The uniqueness properties for pure states, for both UDP and UDA, have also been studied extensively in the case of multipartite quantum systems, where the observables correspond to reduced density matrices (RDMs). That is, the observables are chosen to act nontrivially on only some subsystems. For an $n$-particle system and a constant $k<n$, there are a total of $\left(\begin{array}{l}n \\ k\end{array}\right)$ $k$-RDMs, and the corresponding measurements $\mathbf{A}$ are those $\leqslant k$-body operators. For example, for a three-qubit system and $k=2$, one can choose $\mathbf{A}$ as all the one- and two-particle Pauli operators. Of course, one can also choose to look at some of the $\left(\begin{array}{l}n \\ k\end{array}\right)$-RDMs, rather than all of them. For instance, for a three-particle system, one can look at 2-RDMs of particle pairs $\{1,2\}$ and $\{1,3\}$.

It is known that almost all three-qubit pure states are UDA by their 2-RDMs [11]. These authors also show that UDP implies UDA for three-qubit pure states, for 2-RDMs. This result can be further improved to 2-RDMs of particle pairs $\{1,2\}$ and $\{1,3\}$ [9]. More generally, one can consider a threeparticle system of particles 1,2,3 with Hilbert spaces whose dimensions are $d_{1}, d_{2}, d_{3}$, respectively. If $d_{1} \geqslant d_{2}+d_{3}-1$, then almost all pure states are UDA by their 2-RDMs of particle pairs $\{1,2\}$ and $\{1,3\}$. In contrast, if $d_{1} \geqslant 2$, then almost all pure states are UDP by their 2-RDMs of particle pairs $\{1,2\}$ and $\{1,3\}$, as shown by Diosi [12].

For $n$-particle quantum systems with equal dimensional subsystems, almost all pure states are UDA by their $k$-RDMs of just over half of the parties (i.e., $k \sim n / 2$ ). Furthermore, $\sim n / 2$ properly chosen RDMs among all the $\left(\begin{array}{l}n \\ k\end{array}\right) k$-RDMs suffice [13]. $W$-type states are UDA by their 2-RDMs, and $n-1$ of those 2-RDMs are enough [14]. General symmetric Dicke states are UDA by their 2-RDMs [15]. It has been shown that the only $n$-particle pure states which can not be UDP by their $(n-1)$-RDMs are those GHZ-type states (of the form $\left.\sum_{i} a_{i}|i\rangle^{\otimes n}\right)$, and the result is further improved to the case of UDA [16]. Their results also show that UDP implies UDA for $n$-qubit pure states, for $(n-1)$-RDMs.

Despite these many results, there is no systematic study of these two different types of uniqueness for pure states. This will be the focus of this paper, where we are interested in knowing for given measurements $\mathbf{A}$ whether UDP and UDA are the same or are different. We will give a general argument that there is a gap between the number of observables needed for the two different cases. However, in many interesting circumstances, they can coincide. Our discussions extend naturally to the case of low-rank quantum states instead of just pure states. Here, one can also look at two kinds of uniqueness when measuring given observables A: one is uniqueness among all low-rank states, the other is among all states of any rank.

We organize the paper as follows. In Sec. II, we first show that there is a set of $5 d-7$ observables that ensures every pure state is UDA, which should be compared to the UDP result $4 d-5$. Thus, in general there is a gap between the optimal results for the UDP and UDA cases, and we illustrate this with some examples. Section III discusses the case of observables corresponding to RDMs of a multipartite quantum state, where for the three-particle case, we show that if $d_{1} \geqslant \min \left(d_{2}, d_{3}\right)$, then almost all pure states are UDA by their 2-RDMs of particle pairs $\{1,2\}$ and $\{1,3\}$, improving the bounds given in Ref. [17]. However, this still leaves a gap with the Diosi result for the case of UDP in Ref. [12]. We further discuss circumstances where UDP can imply UDA for all pure states. In Sec. IV, we show that when there are only two independent measurements performed, then UDP always implies UDA by making use of convexity of the numerical range of operators. In a more general case, if there is a compact group of symmetries of the state space which has the span of the operators measured as its set of fixed points, then UDP implies UDA for all pure states. We analyze the possible dimensions for those fixedpoint sets. A summary and some discussions are included in Sec. VI.

\section{NUMBER OF OBSERVABLES FOR UDA}

In this section, we discuss the minimum number of observables needed to have all pure states be UDA. We start by choosing a Hermitian basis $\left\{\lambda_{i}\right\}_{i=0}^{d^{2}-1}$ for the operators on $\mathcal{H}_{d}$. Without loss of generality, we choose $\lambda_{0}=\sqrt{d-1} I$, the identity operator on $\mathcal{H}_{d}$, which has trace $d$. We further require that the $\lambda_{i}$ 's are orthogonal in the sense that, for $i, j \geqslant 0$,

$$
\operatorname{tr} \lambda_{i} \lambda_{j}=d(d-1) \delta_{i j}
$$

The $d \times d$ Hermitian matrices form a real inner-product space with inner product $\langle A, B\rangle=\operatorname{tr}(A B)$, so such a basis $\left\{\lambda_{i}\right\}_{i=0}^{d^{2}-1}$ exists for any dimension $d$. For instance, for the qubit case $(d=2)$, we can choose the Pauli basis

$\lambda_{1}=\left(\begin{array}{ll}0 & 1 \\ 1 & 0\end{array}\right), \quad \lambda_{2}=\left(\begin{array}{cc}0 & -i \\ i & 0\end{array}\right), \quad \lambda_{3}=\left(\begin{array}{cc}1 & 0 \\ 0 & -1\end{array}\right)$.

For the qutrit case $(d=3)$, one can choose $\lambda_{i}=\sqrt{3} M_{i}$ for $i>0$, where $M_{i}$ s are the Gell-Mann matrices given by

$$
\begin{array}{rlrl}
M_{1} & =\left(\begin{array}{lll}
0 & 1 & 0 \\
1 & 0 & 0 \\
0 & 0 & 0
\end{array}\right), & M_{2}=\left(\begin{array}{rrr}
0 & -i & 0 \\
i & 0 & 0 \\
0 & 0 & 0
\end{array}\right), \\
M_{3}=\left(\begin{array}{rrr}
1 & 0 & 0 \\
0 & -1 & 0 \\
0 & 0 & 0
\end{array}\right), & M_{4}=\left(\begin{array}{lll}
0 & 0 & 1 \\
0 & 0 & 0 \\
1 & 0 & 0
\end{array}\right), \\
M_{5}=\left(\begin{array}{rrr}
0 & 0 & -i \\
0 & 0 & 0 \\
i & 0 & 0
\end{array}\right), & M_{6}=\left(\begin{array}{lll}
0 & 0 & 0 \\
0 & 0 & 1 \\
0 & 1 & 0
\end{array}\right), \\
M_{7}=\left(\begin{array}{rrr}
0 & 0 & 0 \\
0 & 0 & -i \\
0 & i & 0
\end{array}\right), & M_{8}=\frac{1}{\sqrt{3}}\left(\begin{array}{rrr}
1 & 0 & 0 \\
0 & 1 & 0 \\
0 & 0 & -2
\end{array}\right) .
\end{array}
$$

For general $d$, one can choose $\lambda_{i}=\sqrt{\frac{d(d-1)}{2}} M_{i}$ for $i>0$, where $M_{i}$ 's are the generalized Gell-Man matrices. We can 
now write any density operator $\rho$ as

$$
\rho=\frac{1}{d}(I+\vec{r} \cdot \vec{\lambda})
$$

where $\quad \vec{\lambda}=\left(\lambda_{1}, \lambda_{2}, \ldots, \lambda_{d^{2}-1}\right), \quad$ and where $\vec{r}=$ $\left(r_{1}, r_{2}, \ldots, r_{d^{2}-1}\right)$ has real entries.

We have $\operatorname{tr} \rho^{2} \leqslant 1$, therefore $\vec{r} \cdot \vec{r} \leqslant 1$, and the equality holds if $\rho$ is a pure state. However, not every state satisfying $\vec{r} \cdot \vec{r}=1$ is a pure state. Indeed, $\rho$ is a pure state if and only if $\rho^{2}=\rho$, which gives equations that $\vec{r}$ needs to satisfy. If one of the observables is a multiple of the identity, then we can drop it from the list of observables without affecting UDA and UDP. If two states agree on an observable $A_{i}$, then they agree on $A_{i}+t I$ for any real scalar $t$, so we can adjust each of the observables $\mathbf{A}=\left(A_{1}, \ldots, A_{m}\right)$ to have trace zero without affecting UDA or UDP. Hence, hereafter we assume all $A_{i}$ are traceless.

For any observable $A_{i}$, we can expand in terms of $\left\{\lambda_{i}\right\}$ as

$$
A_{i}=\sum_{j=1}^{d^{2}-1} \alpha_{i j} \lambda_{j}
$$

Then the average value of $A_{i}$ is given by

$$
\operatorname{tr}\left(A_{i} \rho\right)=\frac{1}{d}\left(d+\sum_{j} r_{j} \alpha_{i j} d(d-1)\right)=1+(d-1) \vec{r} \cdot \vec{\alpha}_{i},
$$

where $\vec{\alpha}_{i}=\left\{\alpha_{i 1}, \alpha_{i 2}, \ldots, \alpha_{i\left(d^{2}-1\right)}\right\}$.

To discuss the problem for any pure state to be UDA, the constant 1 and constant factor $d-1$ can be ignored, as these are the same constants for all states. Therefore, we have

$$
\operatorname{tr}\left(A_{i} \rho\right) \sim \vec{r} \cdot \vec{\alpha}_{i}
$$

where $\sim$ means that the average value of $A_{i}$ for the state $\rho$ is geometrically equivalent to the projection of $\vec{r}$ onto $\vec{\alpha}_{i}$.

Alternatively, define $T: \mathbb{R}^{d^{2}-1} \rightarrow \mathbb{R}^{m}$ by $T(\vec{r})=(\vec{r}$. $\left.\alpha_{1}, \ldots, \vec{r} \cdot \vec{\alpha}_{m}\right)$. Let $L$ be the linear subspace of $\mathbb{R}^{d^{2}-1}$ spanned by $\vec{\alpha}_{1}, \ldots, \vec{\alpha}_{m}$, and let $\pi$ be the orthogonal projection from $\mathbb{R}^{d^{2}-1}$ onto $L$. Then, $\pi$ and $T$ have the same kernel, namely $L^{\perp}$. Thus, for states $\rho_{1}, \rho_{2}$, we have $T\left(\rho_{1}\right)=T\left(\rho_{2}\right)$ if and only if $\pi\left(\rho_{1}\right)=\pi\left(\rho_{2}\right)$, so in considering UDA and UDP we can treat $T$ as being the orthogonal projection onto $L$.

If we subtract the density matrix $I / d$ from all states, then the translated set of states sits in the real $\left(d^{2}-1\right)$-dimensional subspace of trace-zero Hermitian matrices. In this sense, we are actually working with real geometry in $\mathbb{R}^{d^{2}-1}$. All quantum states then sit inside the $\left(d^{2}-1\right)$-dimensional unit ball, with pure states corresponding to unit vectors, but not every vector on the unit $\left(d^{2}-2\right)$-dimensional sphere is a pure state. The observables span an $m$-dimensional subspace that all the quantum states will be projected onto. We will simply say the subspace is spanned by $\mathbf{A}$ when no confusion arises, and we will no longer distinguish an operator $A_{i}$ from the corresponding vector $\vec{\alpha}_{i}$. Indeed, we only consider the real span of $\mathbf{A}$, and we denote it by $\mathcal{S}(\mathbf{A})$. For each $\mathcal{S}(\mathbf{A})$, there is an orthogonal subspace in $\mathbb{R}^{d^{2}-1}$ of dimension $d^{2}-1-m$, which we denote by $\mathcal{S}(\mathbf{A})^{\perp}$. Here, we are taking the orthogonal complement in the space of traceless Hermitian matrices, so that every $V \in \mathcal{S}(\mathbf{A})^{\perp}$ is traceless.

We now are ready to state our first theorem.

Theorem 1. For a $d$-dimensional system $(d>2)$, there exists a set of $5 d-7$ observables for which every pure state is UDA.

To see why this is the case, note that in the above-mentioned geometrical picture, it is clear that a pure state $|\psi\rangle\langle\psi|$ is UDA by measuring $\mathbf{A}$ if there does not exist any operator $V \in \mathcal{S}(\mathbf{A})^{\perp}$, such that $|\psi\rangle\langle\psi|+V$ is positive. One sufficient condition will then be that any operator $V \in \mathcal{S}(\mathbf{A})^{\perp}$ has at least two positive and two negative eigenvalues. We will use this sufficient condition to construct a desired $\mathcal{S}(\mathbf{A})^{\perp}$.

In order to construct $\mathcal{S}(\mathbf{A})^{\perp}$, we provide a set of $m=d^{2}-5 d+6$ linearly independent Hermitian matrices $H_{1}, H_{2}, \ldots, H_{m} \in M_{d}(\mathbb{C})$ explicitly, such that the Hermitian matrix

$$
\sum_{j=1}^{m} r_{j} H_{j}
$$

has at least two positive eigenvalues for any nonzero real vector $r=\left(r_{j}\right) \in \mathbb{R}^{m}$.

Our construction is motivated by and similar to the diagonal filling technique used in Ref. [18], but along the other direction of the diagonals. This then means that measuring $d^{2}-1$ $-\left(d^{2}-5 d+6\right)=5 d-7$ observables is enough for any pure state to be UDA, which proves the theorem. There are indeed technical details to be clarified that we leave to Appendix A.

If we compare our results with those given in Ref. [1], which shows that measuring $4 d-5$ observables are enough for any pure state to be UDP, there exists an obvious gap. We claim that this gap indeed can not be closed in general. To see this, let us look at the simplest case of $d=3$, where the results just compared state that 7 observables are enough for any pure state to be UDP but 8 observables are enough for any pure state to be UDA.

If one can measure a particular set $\mathbf{A}$ with 7 observables and have all pure states be UDA, then also every state also must be UDP for measuring A. According to [1], this only happens if $\mathcal{S}(\mathbf{A})^{\perp}$ contains a single invertible traceless operator $V$, meaning $V$ is rank 3 . Without loss of generality, we can assume the largest eigenvalue $V$ to be positive with an eigenstate $|\psi\rangle$. Then, $|\psi\rangle$ is not UDA by measuring $\mathbf{A}$ since as observed in Ref. [1] there exists a mixed state which also has the same average values as those of $|\psi\rangle$. Therefore, one can not only measure 7 observables for all pure states to be UDA.

For general $d$, our construction needs $5 d-7$ observables. We do not know whether this is the optimal construction, but it is very unlikely one can get this down to $4 d-5$. In other words, in general UDA and UDP for pure states should be indeed two different concepts and there should always be gaps between the number of observables needed to be measured for each case to uniquely determine any pure quantum state. This is one exception, though, which is for the qubit case (i.e., $d=2$ ) where it is shown in Ref. [1] that for all pure states to be UDP, one needs to measure $3=2^{2}-1$ variables, which then uniquely determine any quantum state among all states.

Finally, we remark that our results in Theorem 1 naturally extend to the case of low-rank states. That is, for a rank 
$q<d / 2$ quantum state $\rho$, we can similarly consider two different cases: (1) $\rho$ is uniquely determined by measuring $\mathbf{A}$ among all rank $\leqslant q$ states (which was considered in Ref. [1]), (2) $\rho$ is uniquely determined by measuring $\mathbf{A}$ among all quantum states of any rank.

Theorem 2. For a $d$-dimensional system $(d>2)$ measuring $(4 q+1) d-\left(4 q^{2}+2 q+1\right)$ observables is enough for a rank $\leqslant q$ state to be uniquely determined among all states.

Compared to the results in Ref. [1], where $4 q(d-q)-1$ observables are needed to uniquely determine any rank $\leqslant q$ states among all rank $\leqslant q$ states, when $d$ is large the difference in the leading term has a $d$ gap. The proof idea is similar to that of Theorem 1, so we leave the details to Appendix A.

\section{CASE OF REDUCED DENSITY MATRICES}

In this section, we discuss the case where the Hilbert space $\mathcal{H}_{d}$ is a multipartite quantum system, where the observables correspond to the reduced density matrices (RDMs). That is, the observables are chosen to be acting nontrivially only on some subsystems. For instance, for a three-qubit system, the observables corresponding to the 2-RDMs of particle pairs $\{1,2\}$ can be chosen as

$$
\begin{aligned}
\mathbf{A}= & \left(X_{1}, X_{2}, Y_{1}, Y_{2}, Z_{1}, Z_{2}, X_{1} X_{2}, X_{1} Y_{2}, X_{1} Z_{2}, Y_{1} X_{2}, Y_{1} Y_{2},\right. \\
& \left.Y_{1} Z_{2}, Z_{1} X_{2}, Z_{1} Y_{2}, Z_{1} Z_{2}\right),
\end{aligned}
$$

where $X_{i}, Y_{i}, Z_{i}$ are Pauli $X, Y, Z$ operators acting on the $i$ th qubit.

For simplicity in this section we consider only three-particle systems, labeled by $1,2,3$, and each with Hilbert space dimension $d_{1}, d_{2}, d_{3}$, respectively. That is, $\mathcal{H}_{d}=\mathcal{H}_{d_{1}} \otimes \mathcal{H}_{d_{2}} \otimes \mathcal{H}_{d_{3}}$ and $d=d_{1} d_{2} d_{3}$. Nevertheless, our method naturally extends to systems of more than three particles.

Recall that for a three-particle system, it is known that almost all three-qubit pure states are UDA by their 2-RDMs [11]. This result can be further improved to 2-RDMs of particle pairs $\{1,2\}$ and $\{1,3\}$ [9]. More generally, if $d_{1} \geqslant d_{2}+d_{3}-1$, then almost all pure states are UDA by their 2-RDMs of particle pairs $\{1,2\}$ and $\{1,3\}$ [17]. In contrast, if $d_{1} \geqslant 2$, then almost every pure state is UDP by its $2-\mathrm{RDM}$ of particle pairs $\{1,2\}$ and $\{1,3\}[12]$.

We notice that different from the discussion in Sec. II, one no longer considers uniqueness for all pure states, but 'almost all' of them. This means there exists a measure zero set of pure states which are not uniquely determined. For instance, for the three-qubit case, any state which is local unitarily equivalent to the GHZ-type state

$$
|\mathrm{GHZ}\rangle_{\mathrm{type}}=a|000\rangle+b|111\rangle
$$

can not be UDP, as any state of the form $a|000\rangle+b e^{i \theta}|111\rangle$ has the same 2-RDMs as those of $|\mathrm{GHZ}\rangle_{\text {type }}$. This means that, for a three-qubit pure state $|\psi\rangle$, it is either UDA, or not UDP. In other words, if any three-qubit pure state $|\psi\rangle$ is UDP, then it is UDA by its 2 -RDMs of particle pairs $\{1,2\}$ and $\{1,3\}$. In this sense, we say in this case UDP implies UDA for all pure states. However, for the general case of a three-particle system, there is a gap between known results of UDA and UDP. Our following result improves the bound for the UDA case.
Theorem 3. If $d_{1} \geqslant \min \left(d_{2}, d_{3}\right)$, then almost every tripartite quantum state $|\phi\rangle \in \mathcal{H}_{d_{1}} \otimes \mathcal{H}_{d_{2}} \otimes \mathcal{H}_{d_{3}}$ is UDA by its 2-RDMs of particle pairs $\{1,2\}$ and $\{1,3\}$,

To see why this is the case, an arbitrary pure state $|\phi\rangle$ of this system can be written as

$$
|\phi\rangle_{123}=\sum_{i=1}^{d_{1}} \sum_{j=1}^{d_{2}} \sum_{k=1}^{d_{3}} c_{i j k}|i\rangle_{1}|j\rangle_{2}|k\rangle_{3} .
$$

If there is another state $\rho$ which agrees with $|\phi\rangle$ in its subsystems $\{1,2\}$ and $\{1,3\}$, then we can find a pure state $|\psi\rangle_{1234} \in \mathcal{H}_{d_{1}} \otimes \mathcal{H}_{d_{2}} \otimes \mathcal{H}_{d_{3}} \otimes \mathcal{H}_{d_{4}}$ which agrees with $\rho$ on the subsystem $\{1,2,3\}$ and also agrees with $|\phi\rangle_{123}$ in subsystems $\{1,2\}$ and $\{1,3\}$.

Since the rank of the 2-RDM of the subsystem $\{1,2\}$ is at most $d_{3}$, the pure state $|\psi\rangle_{1234}$ can be written as a superposition of $\left|v_{l}\right\rangle\left|E_{l}\right\rangle$ as follows:

$$
|\psi\rangle_{1234}=\sum_{l=1}^{d_{3}}\left|v_{l}\right\rangle\left|E_{l}\right\rangle
$$

where

$$
\left|v_{l}\right\rangle=\sum_{i=1}^{d_{1}} \sum_{j=1}^{d_{2}} c_{i j l}|i\rangle_{1}|j\rangle_{2}
$$

for any $1 \leqslant l \leqslant d_{3}$. Here, $\left\{\left|E_{l}\right\rangle\right\}_{i=1}^{d_{3}}$ will be vectors (perhaps unnormalized) in $\mathcal{H}_{d_{3}} \otimes \mathcal{H}_{d_{4}}$.

For almost all states $|\phi\rangle$, the set of $\left\{\left|v_{l}\right\rangle\right\}_{i=1}^{d_{3}}$ will be linearly independent. Let us write $\left|E_{l}\right\rangle=\sum_{k=1}^{d_{3}}|k\rangle_{3}\left|e_{l k}\right\rangle_{4}$. For any $1 \leqslant$ $l \leqslant d_{3}$, we will have

$$
|\psi\rangle_{1234}=\sum_{i=1}^{d_{1}} \sum_{j=1}^{d_{2}} \sum_{k, l=1}^{d_{3}} c_{i j l}|i\rangle_{1}|j\rangle_{2}|k\rangle_{3}\left|e_{l k}\right\rangle_{4} .
$$

Now let us consider the subsystem $\{1,3\}$. Since $|\phi\rangle_{123}$ and $|\psi\rangle_{1234}$ have the same RDMs for particles $\{1,3\}$, this gives

$$
\operatorname{tr}_{2}|\phi\rangle\left\langle\phi\left|=\operatorname{tr}_{\{2,4\}}\right| \psi\right\rangle\langle\psi| \text {. }
$$

Substituting Eqs. (16) and (17) into Eq. (20), and comparing each matrix element, results in the following equalities (for all $\left.m, m^{\prime}, n, n^{\prime}\right)$ :

$$
\sum_{j=1}^{d_{2}} c_{m j n} c_{m^{\prime} j n^{\prime}}^{*}=\sum_{j=1}^{d_{2}} \sum_{k, k^{\prime}=1}^{d_{3}} c_{m j k} c_{m^{\prime} j k^{\prime}}^{*}\left\langle e_{k^{\prime} n^{\prime}} \mid e_{k n}\right\rangle .
$$

Now, let us define $x_{i j k l}=\left\langle e_{i j} \mid e_{k l}\right\rangle$. Then, Eq. (21) is a linear equation system with variables $x_{i j k l}$. It is not hard to verify that

$$
x_{i j k l}= \begin{cases}1 & \text { if } i=j, k=l \\ 0 & \text { otherwise }\end{cases}
$$

is a solution to the equation system, which corresponds to the state $|\phi\rangle_{123}$.

Now, we need to show that when $d_{1} \geqslant \min \left\{d_{2}, d_{3}\right\}$, Eq. (21) has only one solution which is given by Eq. (22). It turns out that this is indeed the case which then proves Theorem 3 . In fact, the linear equations above are generically linearly independent. To see this, let us fix $n, n^{\prime}$ and $m, m^{\prime}$, the right-hand side of Eq. (21) is $\sum_{k, k^{\prime}=1}^{d_{3}}\left\langle\left.\alpha\right|_{m^{\prime} k^{\prime}} \mid \alpha\right\rangle_{m k} x_{k^{\prime} n^{\prime} k n}$ where 
$|\alpha\rangle_{m k}=\sum_{j=1}^{d_{2}} c_{m j k}|j\rangle$. Then, the coefficient matrix can be written as the following:

$$
\left(\begin{array}{cccc}
\left\langle\left.\alpha\right|_{11} \mid \alpha\right\rangle_{11} & \left\langle\left.\alpha\right|_{11} \mid \alpha\right\rangle_{12} & \ldots & \left\langle\left.\alpha\right|_{1 d_{3}} \mid \alpha\right\rangle_{1 d_{3}} \\
\left\langle\left.\alpha\right|_{11} \mid \alpha\right\rangle_{21} & \left\langle\left.\alpha\right|_{11} \mid \alpha\right\rangle_{22} & \ldots & \left\langle\left.\alpha\right|_{1 d_{3}} \mid \alpha\right\rangle_{2 d_{3}} \\
\vdots & \vdots & \ddots & \vdots \\
\left\langle\left.\alpha\right|_{d_{1} 1} \mid \alpha\right\rangle_{d_{1} 1} & \left\langle\left.\alpha\right|_{d_{1} 1} \mid \alpha\right\rangle_{d_{1} 2} & \ldots & \left\langle\left.\alpha\right|_{d_{1} d_{3}} \mid \alpha\right\rangle_{d_{1} d_{3}}
\end{array}\right)
$$

The $\left[d_{1}(i-1)+j, d_{3}(p-1)+q\right]$ entry in the above matrix is $\left\langle\left.\alpha\right|_{i p} \mid \alpha\right\rangle_{j q}$.

If there is more than one solution, then the determinant of the above matrix should be zero. Note that the determinant can be written as a polynomial of $c_{m j k}$ 's and $c_{m^{\prime} j k^{\prime}}^{*}$ 's. Since $\prod c_{i i i}$ appears only once in the polynomial, the determinant of the top $d_{3}^{2}$ by $d_{3}^{2}$ submatrix must be nonzero generically. Therefore, $d_{1}^{2} d_{3}^{2}$ linear equations are sufficient to determine $d_{3}^{4}$ variables.

However, we do not know whether the sufficient condition given by Theorem 3 for almost all three-particle pure states to be UDA by its 2 -RDMs of particle pairs $\{1,2\}$ and $\{1,3\}$ is also necessary. This still leaves a gap between the result of Theorem 3 for UDA, and the result for UDP in [12]. They both only coincide when $d_{1}=d_{2}=d_{3}=2$, i.e., the three-qubit case. It remains open for other cases, whether UDP can imply UDA.

Following a similar discussion as in Sec. II, our result in this section also extends to uniqueness of low-rank quantum states. In particular, we have the following theorem.

Theorem 4. Almost every tripartite density operator $\rho$ acting on the Hilbert space $\mathcal{H}_{d_{1}} \otimes \mathcal{H}_{d_{2}} \otimes \mathcal{H}_{d_{3}}$ with rank no more than $\left\lfloor\frac{d_{1}}{d_{3}}\right\rfloor$ can be uniquely determined among all states by its 2-RDMs of particle pairs $\{1,2\}$ and $\{1,3\}$.

This theorem establishes a unique determination result for low-rank mixed states given their RDMs. The proof is a direct extension of that for Theorem 3, but with more lengthy details that we will include in Appendix B.

Let us look at some consequences of Theorem 4. Consider a four-qubit system with qubits 1,2,3,4, and look at the qubits 1,2 as a single systems $1^{\prime}$. Then, Theorem 4 says also that almost all four qubit states of rank 2 are UDA by their RDMs of particles $\left\{1^{\prime}, 3\right\}=\{1,2,3\}$ and $\left\{1^{\prime}, 4\right\}=\{1,2,4\}$, or one can say that almost all four qubit states of rank 2 are UDA by their 3-RDMs. This is indeed consistent with the multipartite result in Ref. [13] which states that almost all four-qubit pure states are UDA by their 3-RDMs, and our result is indeed stronger. This demonstrates that our analysis naturally extends to systems of more than three particles. We also remark that the rank of a state $\rho$ which could be UDA by its $k$-RDMs needs to be relatively low, otherwise one can always find another state $\rho^{\prime}$ with lower rank which has the same $k$-RDMs as those of $\rho$ [19].

Finally, we remark that for the three-qubit case, the GHZtype states and all their local unitary equivalents are the only states that are UDA by their 2-RDMs. One may wonder for the systems discussed in Theorems 3 and 4 as to which states are undetermined. Unfortunately, it is not easy to give a complete characterization of these undetermined states, as methods used in the proof of Theorems 3 and 4 do not directly give that. However, what we do know is that there are certainly states beyond the generalized GHZ type, i.e., $\sum_{i=1}^{\min \left(d_{1}, d_{2}, d_{3}\right)} \alpha_{i}|i i i\rangle$, or their local unitary equivalents, which are undetermined.

As an example, consider a graph state $\left|\psi_{1}\right\rangle$ of five qubits which is stabilized by the stabilizer group with generators

$$
\begin{array}{ll}
g_{1}=X_{1} Z_{2} Z_{5}, & g_{2}=Z_{1} X_{2} Z_{3}, \quad g_{3}=Z_{2} X_{3} Z_{4}, \\
g_{4}=Z_{3} X_{4} Z_{5}, & g_{5}=Z_{1} Z_{4} X_{5} .
\end{array}
$$

Let us consider this as a three-particle system with qubits $\{1,2\}$ the first particle, qubits $\{3,4\}$ the second particle, and qubit $\{5\}$ the third particle. This is then a three-particle system with different local dimensions satisfying the dimension condition given by Theorem 3 . Note that $\left|\psi_{1}\right\rangle$ is undetermined by the RDMs of particles $\{1,2,3,4\}$ and $\{1,2,5\}$. This is because that the state $\left|\psi_{2}\right\rangle$ which is stabilized by the stabilizer group with generators $\left\{g_{1}, g_{2}, g_{3}, g_{4},-g_{5}\right\}$ has the same RDMs of particles $\{1,2,3,4\}$ and $\{1,2,5\}$ as those of $\left|\psi_{1}\right\rangle$. Furthermore, $\left|\psi_{1}\right\rangle$ has RDMs of rank 4 for both the first and second particles, and RDM of rank 2 for the third particle, which certainly is not local unitary equivalent to the generalized GHZ-type states.

\section{CASE OF ONLY TWO OBSERVABLES}

In Secs. II and III, we discussed the difference and coincidence between the two kinds of uniqueness for pure states UDA and UDP, which in general are not the same thing. However, in certain interesting circumstances such as the three-qubit case with respect to 2-RDMs, and in general the $n$-qubit case with respect to $(n-1)$-RDMs, they do coincide. Starting from this section we would like to build some general understanding of the circumstances when UDP implies UDA for all pure states.

We start from the simplest case of $m=2$, where only two observables are measured, i.e., $\mathbf{A}=\left(A_{1}, A_{2}\right)$. Intuitively, in this extreme case almost no pure state can be uniquely determined, either UDA or even UDP. However, there are also exceptions. For instance, if one of the observables, say $A_{1}$, has a nondegenerate ground state $|\psi\rangle$, then $|\psi\rangle$ is UDA (hence, of course, UDP) even by measuring $A_{1}$ only. One would hope this is the only exception, that is, for a pure state $|\psi\rangle$, either it is UDA, or it is not UDP, when only two observables are measured. We make this intuition rigorous by the following theorem.

Theorem 5. When only two observables are measured, i.e., $\mathbf{A}=\left(A_{1}, A_{2}\right)$, UDP implies UDA for any pure state $|\psi\rangle$, regardless of the dimension $d$.

To prove this theorem, recall that measuring $\mathbf{A}$ (i.e., measuring every observable in $\mathbf{A}$ ) for all quantum states $\rho$ returns the set $C_{m}(\mathbf{A})$ given by Eq. (3). We know that $C_{m}(\mathbf{A})$ is a convex set, meaning for any $\vec{x}, \vec{y} \in C_{m}(\mathbf{A})$, we have $(1-s) \vec{x}+s \vec{y} \in C_{m}(\mathbf{A})$ for any $0<s<1$.

For pure states, the corresponding set of average values is given by $W_{m}(\mathbf{A})$ as defined in Eq. (5). Unlike $C_{m}(\mathbf{A}), W_{m}(\mathbf{A})$ in general is not convex. Nevertheless, it is easy to see that $W_{m}(\mathbf{A})=C_{m}(\mathbf{A})$ when $W_{m}(\mathbf{A})$ is convex.

For $m=2$, the Hausdorff-Toeplitz theorem [20,21] gives convexity of the numerical range of any operator, which in turn shows that $W_{2}(\mathbf{A})$ is convex. We explain it briefly here. For any operator $B$ acting on a Hilbert space $\mathcal{H}_{d}$, the numerical 
range of $B$ is the set of all complex numbers $\langle\psi|B| \psi\rangle$, where $|\psi\rangle$ ranges over all pure states in $\mathcal{H}_{d}$.

Note that one can always write $B$ as

$$
\begin{aligned}
B & =\frac{1}{2}\left[\left(B+B^{\dagger}\right)+\left(B-B^{\dagger}\right)\right] \\
& =\frac{1}{2}\left[\left(B+B^{\dagger}\right)+i\left(-i B+i B^{\dagger}\right)\right] .
\end{aligned}
$$

If we define $A_{1}:=\left(B+B^{\dagger}\right) / 2$ and $A_{2}:=\left(-i B+i B^{\dagger}\right) / 2$, then clearly both $A_{1}$ and $A_{2}$ are Hermitian. Then, $W_{2}(\mathbf{A})$ is nothing but the numerical range of $B=A_{1}+i A_{2}$ and hence is convex.

Furthermore, by studying the properties of the numerical range, it was shown in Ref. [22] (using different terminology) that if a pure state $|\psi\rangle$ is UDP, the point $\vec{x}:=\mathbf{A}(|\psi\rangle)$ must be an extreme point of $W_{2}(\mathbf{A})$. Here, $\vec{x}$ is an extreme point of the convex set $W_{2}(\mathbf{A})$ if there do not exist $\vec{y}, \vec{z} \in W_{2}(\mathbf{A})$, such that $\vec{x}=(1-s) \vec{y}+s \vec{z}$ for some $0<s<1$.

Because $W_{2}(\mathbf{A})=C_{2}(\mathbf{A}), \vec{x}$ is also an extreme point of $C_{2}(\mathbf{A})$. One can further show that for any extreme point $\vec{x}$ of $C_{2}(\mathbf{A})$, and any quantum state $\rho$ with $\mathbf{A}(\rho)=\vec{x}$, any pure quantum state $|\phi\rangle$ in the range of $\rho$ will also have $\mathbf{A}(|\phi\rangle)=\vec{x}$. This then implies that if a pure state $|\psi\rangle$ is UDP by measuring A, it must also be UDA, which proves the theorem. Again, all the technical details of the proof will be presented in Appendix C.

In an attempt to extend Theorem 5 to the $m \geqslant 3$ case, a natural question that one could ask is whether or not UDP implies UDA whenever $W_{m}(\mathbf{A})$ is convex. Unfortunately, this is not the case, as demonstrated by the following example.

For the qutrit case $(d=3)$, consider the observables $\mathbf{A}=$ $\left(M_{1}, M_{2}, M_{3}\right)$, where the $M_{i}$ 's are the Gell-Mann matrices given in Eq. (9). These are the Pauli operators embedded in the qutrit space. It is easily verified that in this case, $W_{m}(\mathbf{A})$ is the Bloch sphere together with its interior and is thus convex. Nonetheless, the unique pure state compatible with measurement result $(0,0,0)$ is the state $|2\rangle$, even though there are many mixed states sharing this measurement result, such as $\frac{1}{2}(|0\rangle\langle 0|+| 1\rangle\langle 1|)$.

Therefore, although the Hausdorff-Toeplitz theorem Refs. [20,21] is famous for showing the convexity of numerical range of any operator, there is indeed a deeper reason than just the convexity of the numerical range which governs the validity of Theorem 5. We leave the more detailed discussion to Appendix C.

\section{SYMMETRY OF THE STATE SPACE}

In this section, we discuss some circumstances where UDP implies UDA in a more general context where more than two observables are measured, i.e., $m>2$. Our focus is on the symmetry of the set of all quantum states. For a $d$-dimensional Hilbert space $\mathcal{H}_{d}$ we denote this set of states by $K_{d}$, that is,

$$
K_{d}=\left\{\rho \mid \rho \text { acts on } \mathcal{H}_{d}, \operatorname{tr}(\rho)=1\right\} .
$$

Note that $K_{d}$ is convex, as we know that for any $\rho_{1}, \rho_{2} \in$ $K_{d},(1-s) \rho_{1}+s \rho_{2} \in K_{d}$ for all $0<s<1$. Furthermore, the extreme points of $K_{d}$ are all the pure states. $K_{d}$ is also called the state space for all the operators acting on $\mathcal{H}_{d}$.

We now explain the intuition. If $K_{d}$ has a certain symmetry, then two pure states $\left|\psi_{1}\right\rangle$ and $\left|\psi_{2}\right\rangle$ that are "connected" by the

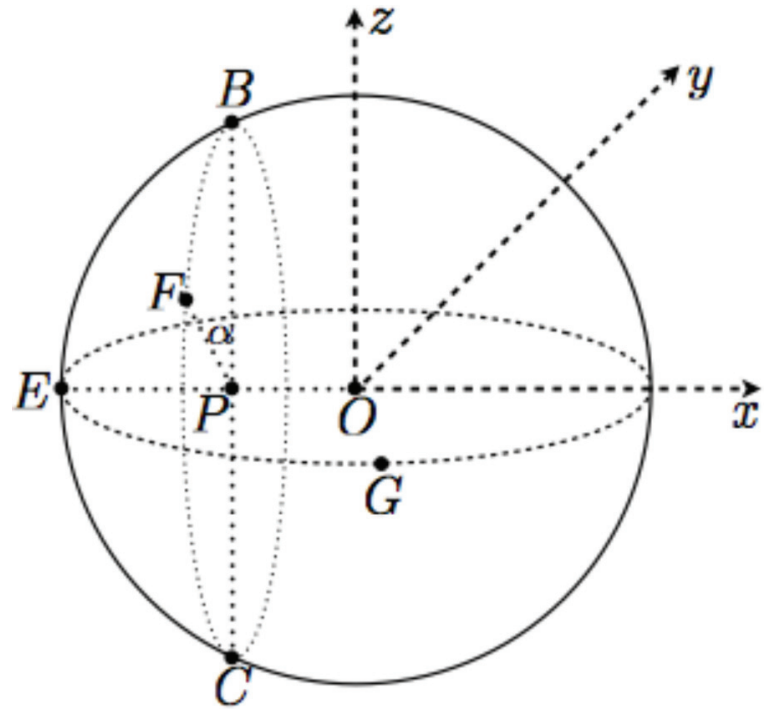

FIG. 1. Symmetry of the Bloch ball.

symmetry will give the same measurement results, and states $|\psi\rangle$ fixed by the symmetry will also be fixed by the projection onto the space of observables. In this situation, UDP implies UDA for all pure states.

To make this intuition concrete, let us first consider an example for $d=2$, i.e., the qubit case. We know that $K_{d}$ can be parametrized as in Eq. (10), where for $d=2, \lambda_{1}=X, \lambda_{2}=$ $Y, \lambda_{3}=Z$ are chosen as Pauli matrices given in Eq. (8). Here, $K_{d}$ is the Bloch ball as shown in Fig. 1. The Bloch ball is clearly a convex set and the extreme points are those pure states on the boundary, which give the Bloch sphere.

We know that geometrically, measuring the observables in A corresponds to the projection onto the plane spanned by $\mathbf{A}$. For example, if we measure the Pauli $X$ and $Y$ operators, then geometrically this corresponds to the projection of the Bloch ball onto the $x y$ plane. Since the Bloch ball has reflection symmetry with respect to the $x y$ plane, two pure states (e.g., points $B$ and $C$ ) connected by that symmetry will project onto the same measurement result $P$, as will all mixtures of $B$ and $C$. Hence, neither UDP nor UDA hold for such pure states for measuring $X$ and $Y$. On the other hand, pure states fixed by the reflection symmetry are also fixed by the projection onto the $x y$ plane. These are precisely the points on the Bloch sphere that are in the $x y$ plane (e.g., the points $E$ and $G$ in Fig. 1), and for such pure states both UDP and UDA hold. Therefore, for the observables $X, Y$ we conclude that UDP $=$ UDA.

Now, let us look at another case where we only measure the Pauli $X$ operator. Consider the group of symmetries of the Bloch ball consisting of rotation around the $x$ axis. (Rotation by angle $\alpha$ is shown in Fig. 1. In that figure, point $B$ will become point $F$ after this particular rotation, and indeed both points $B$ and $F$ yield the same measurement result, which is represented by point $P$ on the $x$ axis.) Note that two points on the Bloch sphere will project to the same measurement result on the $x$ axis if and only if they are in the same orbit under the rotation group. Thus, a measurement result will come from a single pure state exactly when that pure state is a fixed point, and hence either both or neither of UDP and UDA hold for each pure state. For example, the point $E$ is fixed by the rotation, and is uniquely 
determined by the measurement of $X$ among all states. $E$ corresponds to the -1 eigenstate of the Pauli $X$ operator. Therefore, the rotational symmetry of the Bloch ball along the $x$ axis gives UDP $=$ UDA for any pure state when measuring the Pauli $X$ operator, which corresponds to the $x$ axis.

Mathematically, a symmetry of $K_{d}$ is an affine automorphism of $K_{d}$. If $U \in M_{d}$ is unitary, the map taking $\rho$ to $U \rho U^{\dagger}$ is such an affine automorphism (which for $d=2$ will just be rotation around some axis of the Bloch ball). For instance, the rotation symmetry along the $x$ axis by an angle $\alpha$ is given by conjugation by the unitary operator $\exp (-i X \alpha / 2)$. If $V$ is the conjugate linear map given by complex conjugation in the computational basis $\left(V|\psi\rangle=\left|\psi^{*}\right\rangle\right)$, then the map taking $\rho$ to $V \rho V^{\dagger}$ is the transpose map. For $d=2$, this map is reflection of the Bloch ball in the $x y$ plane.

Recall that for a set of observables $\mathbf{A}$, we denote the real linear span by $\mathcal{S}(\mathbf{A})$. When discussing the uniqueness problems, it makes no difference if we append the identity operator to $\mathbf{A}$. Let us then assume $\mathbf{A}=\left(I, A_{1}, \ldots, A_{m}\right)$. We are now ready to put our intuition into a theorem.

Theorem 6. Assume there exists a compact group $G$ of affine automorphisms of $K_{d}$ whose fixed point set is $K_{d} \bigcap S(\mathbf{A})$. Then, each pure state acting on $\mathcal{H}_{d}$ which is UDP for measuring A is also UDA.

In the first example above, the group for the reflection consists of the two-element group generated by the reflection. In the rotation example, we can take the group to consist of all rotations around the given axis. We will leave the detailed mathematical proof of Theorem 6 to Appendix D, where operator algebras are one ingredient of the proof.

To motivate some further consequences of Theorem 6 , consider a simple example. If $\mathbf{A}$ consists of a basis of diagonal matrices (i.e., a set of mutually commuting observables), then for any pure state, UDP implies UDA by Theorem 6. Here, the group of symmetries can be taken to be conjugation by all diagonal unitaries. This group has fixed-point set $K_{d} \bigcap S(\mathbf{A})$. In a more general case, if the complex span of $S(\mathbf{A})$ is a *-subalgebra of the operators acting on $\mathcal{H}_{d}$, then UDP $=$ UDA for all pure states for measuring $\mathbf{A}$. This is a natural corollary of Theorem 6 that we will also discuss in detail in Appendix D.

\section{CONCLUSION AND DISCUSSION}

In this work, we have discussed the uniqueness of quantum states compatible with given results for measuring a set of observables. For a given pure state, we consider two different types of uniqueness, UDP and UDA. We have taken the first step to study their relationship systematically. In doing so, we have established a number of results, but also leave with many open questions.

First of all, although in general UDP and UDA are evidently different concepts, their difference is surprisingly "not that large." Specifically in the sense of general counting of the number of variables one needs to measure to uniquely determine all pure states in a $d$-dimensional Hilbert space. Compared to full quantum tomography which requires $d^{2}-1$ variables measured to uniquely determine any quantum state, the $5 d-7$ observables we have constructed to uniquely determine any pure state among all states are a significant improvement. It is indeed larger than the $4 d-5$ observables given in Ref. [1] to uniquely determine any pure state among all pure states, but the difference is only linear in $d$. We do not know whether there could be another construction for which we could further close the linear difference between UDA and UDP, to leave only a constant gap for large $d$.

When the Hilbert space is a multipartite quantum system, and the observables correspond to the RDMs, we focused on the situation when "almost all pure states" are uniquely determined. We considered a three-particle system with Hilbert space $\mathcal{H}_{d}=\mathcal{H}_{d_{1}} \otimes \mathcal{H}_{d_{2}} \otimes \mathcal{H}_{d_{3}}$, and showed that if $d_{1} \geqslant \min \left(d_{2}, d_{3}\right)$, then almost all pure states are UDA by their 2 -RDMs of particle pairs $\{1,2\}$ and $\{1,3\}$. This improves the results of [17], where $d_{1} \geqslant d_{2}+d_{3}-1$ is required; however, it still leaves a gap compared to the Diosi UDP result which states that for $d_{1} \geqslant 2$, almost all pure states are UDP by their 2 -RDMs of particle pairs $\{1,2\}$ and $\{1,3\}$. Because our proof only gives a sufficient condition for UDA, we do not know whether it can be further improved. We also do not have an example showing there is indeed a gap between UDA and UDP for almost all three-particle pure states to be uniquely determined by 2 -RDMs of particle pairs $\{1,2\}$ and $\{1,3\}$.

Finally, we considered situations for which we can show that UDP implies UDA. These include (i) the general twoqubit system; (ii) the three-qubit system when we consider uniqueness for almost all pure states and the measurements correspond to 2-RDMs; (iii) when only two observables are measured; and (iv) the observables measured correspond to some symmetry of the state space. However, we do not know how far we are from enumerating all the possible situations that UDP implies UDA, when considering uniqueness for all pure states or almost all pure states. In principle, one can even consider the relationship between UDP and UDA for special subsets of pure states.

We believe our systematic study of the uniqueness of quantum states compatible with given measurement results sheds light on several aspects of quantum information theory and its connection to different topics in mathematics. These include quantum tomography and the space of Hermitian operators, unique ground states of local Hamiltonians and general solutions to certain linear equations, measurements and numerical ranges of operators, and the geometric meaning of measurements and the symmetry of state space. We thus conclude with several open questions that we believe warrant further investigation.

\section{ACKNOWLEDGMENTS}

J.C. is supported by NSERC and NSF of China (Grant No. 61179030). Z.J. acknowledges support from NSERC, ARO. N.J. is supported by the University of Guelph Brock Scholarship and an NSERC Postdoctoral Fellowship. D.W.K. is supported by NSERC Discovery Grant No. 400160 and NSERC Discovery Accelerator Supplement No. 400233. B.Z. is supported by NSERC and CIFAR.

\section{APPENDIX A: PROOF OF THEOREMS 1 AND 2}

Theorem 1 can be implied by the following lemma.

Lemma 1. There exists a set of $m=d^{2}-5 d+6$ linearly independent Hermitian matrices $H_{1}, H_{2}, \ldots, H_{m} \in M_{d}(\mathbb{C})$, 
such that the Hermitian matrix

$$
\sum_{j=1}^{m} r_{j} H_{j}
$$

has at least two positive eigenvalues for any nonzero real vector $r=\left(r_{j}\right) \in \mathbb{R}^{m}$.

Proof. We prove the statement by giving an explicit construction. Our proof is motivated by and similar to the diagonal filling technique used in Ref. [18], but along the other direction of the diagonals.

We will need the Lemma 9 from Ref. [18] about totally nonsingular matrix, which we restate as Lemma 3 in the following. For simplicity, we also assume that the totally nonsingular matrix is real. Therefore, for any length $L \in \mathbb{N}$ and $L \geqslant 2$, there is $L-1$ linearly independent real vectors such that every nonzero linear combination of them has at least two nonzero entries.

Let $H=\left(H_{j j^{\prime}}\right) \in M_{d}(\mathbb{C})$ be a matrix. We will always fix the diagonal to be zero, namely, $H_{j j}=0$ for $0 \leqslant j \leqslant d-$ 1. In the upper triangular part of the matrix not including the diagonal, there are $2 d-3$ lines of entries parallel to the antidiagonal. That is, each line contains entries $H_{j j^{\prime}}$ with $j<$ $j^{\prime}$ and $j+j^{\prime}=k$ where $k$ goes from 1 to $2 d-3$. We will call it the $k$ th line of the matrix in the following. We also call the set of entries $H_{j j^{\prime}}$ with $j+j^{\prime}=k$ the $k$ th antidiagonal. It is easy to see that the length $L_{k}$ of the $k$ th line is

$$
L_{k}= \begin{cases}{\left[\frac{k+1}{2}\right]} & \text { for } k \leqslant d-1, \\ {\left[\frac{2 d-1-k}{2}\right]} & \text { otherwise. }\end{cases}
$$

So the length $L_{k} \geqslant 2$ for $3 \leqslant k \leqslant 2 d-5$, and we can find $L_{k}-1$ real vectors for which every nonzero linear combination has at least two nonzero entries. For each of the $L_{k}-1$ vectors, we can form two Hermitian matrices. One of them is the symmetric one whose $k$ th line is filled with the vector, and the lower triangular part determined by the Hermitian condition. Such a matrix is a real symmetric matrix having nonzero entries only on the $k$ th antidiagonal. We will call it a real $k$ th line matrix. The other is the one with $k$ th line filled with the vector multiplied by $i=\sqrt{-1}$, and the lower part is determined by the Hermitian condition. This is a matrix consisting of purely imaginary entries on the $k$ th antidiagonal and we call it an imaginary $k$ th line matrix.

Now, we prove that the constructed matrices satisfy our requirement. First, we prove that the matrices are linearly independent. It suffices to show that the matrices of the nonzero $k$ th line is linearly independent. Let $\left\{v_{j}\right\}$ be the set of linearly independent real vectors chosen for the $k$ th line. We need to show that $\left\{\left(v_{j}, v_{j}\right),\left(i v_{j},-i v_{j}\right)\right\}$ is linearly independent over $\mathbb{C}$. If the contrary is true, that is, there exists complex numbers $c_{j}, d_{j}$ not all zero such that

$$
\sum_{j} c_{j}\left(v_{j}, v_{j}\right)+\sum_{j} d_{j}\left(i v_{j},-i v_{j}\right)=0 .
$$

This is equivalent to

$$
\sum_{j} c_{j} v_{j}+i \sum_{j} d_{j} v_{j}=0, \quad \sum_{j} c_{j} v_{j}-i \sum_{j} d_{j} v_{j}=0 .
$$

From the above two equations, we get $\sum_{j} c_{j} v_{j}=0$ and $\sum_{j} d_{j} v_{j}=0$, which is a contradiction.

Next, we prove that for any nonzero real coefficient $r \in \mathbb{R}^{m}$, the matrix $H=\sum r_{j} H_{j}$ has at least two positive eigenvalues. Let $k_{0}$ be the largest $k$ such that there is a $k$ th line matrix $H_{j}$ whose coefficient $r_{j}$ is nonzero. Then, either the real $k_{0}$ th line matrices or the imaginary ones have nonzero coefficients. By the construction, this implies that there are at least two nonzero entries on the $k_{0}$ th line of the matrix $H$. Let the nonzero entries be $a, b \in \mathbb{C}$. We then have a principle submatrix of $H$ that has the form

$$
\left(\begin{array}{llll}
0 & x & y & a \\
\bar{x} & 0 & b & 0 \\
\bar{y} & \bar{b} & 0 & 0 \\
\bar{a} & 0 & 0 & 0
\end{array}\right),
$$

where $x, y$ are two unknown numbers and $\bar{a}$ represents the complex conjugate of $a$. This matrix has trace 0 and determinant $|a b|^{2}$. Therefore, it has exactly two positive eigenvalues. As it is a principle submatrix of matrix $H$, follows from Theorem 7, $H$ has at least two positive eigenvalues.

The number of matrices thus constructed is the summation

$$
m=\sum_{k=3}^{2 d-5} 2\left(L_{k}-1\right)
$$

which can be computed to be

$$
d^{2}-5 d+6
$$

Discussion. We note that our construction will also imply that the matrix has at least two negative eigenvalues, thus at least rank 4 . But, our bound is even better than the $(d-3)^{2}$ bound on the dimension of subspaces in which every matrix has rank $\geqslant 4$. This is not a contradiction as we are considering all real combinations. For example, the case of $d=4$ has two matrices for our purpose, namely,

$H_{1}=\left(\begin{array}{llll}0 & 0 & 0 & 1 \\ 0 & 0 & 1 & 0 \\ 0 & 1 & 0 & 0 \\ 1 & 0 & 0 & 0\end{array}\right)$ and $H_{2}=\left(\begin{array}{rrrr}0 & 0 & 0 & i \\ 0 & 0 & i & 0 \\ 0 & -i & 0 & 0 \\ -i & 0 & 0 & 0\end{array}\right)$.

These two matrices do satisfy our requirements, but their span over $\mathbb{C}$ contains a rank-2 matrix $\mathrm{H}_{1}+i \mathrm{H}_{2}$.

Generalization. Similarly, length $L_{k} \geqslant q+1$ for $2 q+1 \leqslant$ $k \leqslant 2 d-2 q-3$, and we can find $L_{k}-q$ real vectors for which every nonzero linear combination has at least $q+1$ nonzero entries. For each of the $L_{k}-q$ vectors, we can also form two Hermitian matrices. Such constructed matrices are linearly independent and any real linear combination has at least $q+1$ positive eigenvalues.

We restate our result as Lemma 2 which will lead to Theorem 1.

Lemma 2. There exists a set of $m=d^{2}-(4 q+$ $1) d+\left(4 q^{2}+2 q\right)$ linearly independent Hermitian matrices $H_{1}, H_{2}, \ldots, H_{m} \in M_{d}(\mathbb{C})$, such that the Hermitian matrix

$$
\sum_{j=1}^{m} r_{j} H_{j}
$$


has at least $q+1$ positive eigenvalues for any nonzero real vector $r=\left(r_{j}\right) \in \mathbb{R}^{m}$.

We just follow the lines of the proof of Lemma 1. To complete our argument, we need to show that any $2(q+1)$ by $2(q+1)$ invertible traceless, Hermitian, upper left triangular matrix has exactly $q+1$ positive eigenvalues.

Let us prove this claim by induction. When $q=1$, it is already known. Let us assume this claim holds true for any $q \leqslant r$. Then, for $q=r+1$, we can write such matrix $A$ in the following form:

$$
\left(\begin{array}{ccccccc}
0 & x_{1} & x_{2} & \ldots & x_{2 r+1} & x_{2 r+2} & a \\
\overline{x_{1}} & 0 & y_{1} & \ldots & y_{2 r} & b & 0 \\
\overline{x_{2}} & \overline{y_{1}} & 0 & \ldots & c & 0 & 0 \\
\vdots & \vdots & \vdots & \vdots & \vdots & \vdots & \\
\bar{a} & 0 & 0 & 0 & 0 & 0 & 0
\end{array}\right) .
$$

One may observe that, by deleting the first and the last rows and columns, we will have a $2 r \times 2 r$ invertible, traceless, Hermitian, upper left triangular submatrix.

From our assumption, this submatrix has exactly $r$ positive eigenvalues which means $A$ has at least $r$ positive eigenvalues and at least $r$ negative eigenvalues. Note that its determinant equals to $(-1)^{q+1}|a b \ldots|^{2}$. This follows that $A$ has exactly $r+2$ positive eigenvalues which completes our argument.

The number of matrices thus constructed is the summation

$$
\begin{aligned}
m= & 2 \sum_{k=2 q+1}^{2 d-2 q-3}\left(L_{k}-q\right) \\
= & 2 \sum_{k=2 q+1}^{d-1}\left(\left[\frac{k+1}{2}\right]-q\right) \\
& +2 \sum_{k=d}^{2 d-2 q-3}\left(\left[\frac{2 d-1-k}{2}\right]-q\right) \\
= & d^{2}-(4 q+1) d+\left(4 q^{2}+2 q\right) .
\end{aligned}
$$

Lemma 3 (Lemma 9, Ref. [18]). Let $M$ be a $d \times d$ totally nonsingular matrix, with $d \geqslant n$. Let $v$ be any linear combination of $n$ of the columns of $M$. Then $v$ contains at most $n-1$ zero elements.

Theorem 7 (Theorem 4.3.15, [23]). Let $A$ be a $n \times n$ Hermitian matrix, let $r$ be an integer with $1 \leqslant r \leqslant n$, and let $A_{r}$ denote any $r \times r$ principle submatrix of $A$ (obtained by deleting $n-r$ rows and the corresponding columns from $A$ ). For each integer $k$ such that $1 \leqslant k \leqslant r$ we have

$$
\lambda_{k}^{\uparrow}(A) \leqslant \lambda_{k}^{\uparrow}\left(A_{r}\right) \leqslant \lambda_{k+n-r}^{\uparrow}(A) .
$$

\section{APPENDIX B: PROOF OF THEOREM 4}

Theorem 8. Almost every tripartite density operator $\rho \in$ $\mathcal{B}\left(\mathcal{H}_{d_{1}} \otimes \mathcal{H}_{d_{2}} \otimes \mathcal{H}_{d_{3}}\right)$ with rank no more than $\left\lfloor\frac{d_{1}}{d_{3}}\right\rfloor$ is UDA by its 2-RDMs of particle pairs $\{1,2\}$ and $\{1,3\}$.

Proof. For any $\rho_{123} \in \mathcal{B}\left(\mathcal{H}_{d_{1}} \otimes \mathcal{H}_{d_{2}} \otimes \mathcal{H}_{d_{3}}\right)$, we can choose $|\phi\rangle_{1234}$ to be the pure state whose 3-RDM of particles $\{1,2,3\}$ is exactly $\rho_{123}$. We can further assume $d_{4} \leqslant \operatorname{rank} \rho$. Let us write

$$
|\phi\rangle_{1234}=\sum_{i_{1}, i_{2}, i_{3}, i_{4}} \lambda_{i_{1} i_{2} i_{3} i_{4}}\left|i_{1}\right\rangle_{1}\left|i_{2}\right\rangle_{2}\left|i_{3}\right\rangle_{3}\left|i_{4}\right\rangle_{4}
$$

If there is another $\sigma_{123}$ agrees with $\rho_{123}$ in subsystems $\{1,2\}$ and $\{1,3\}$. Then, we can find some pure state $|\psi\rangle_{1235}$ whose 3 -RDM of particle set $\{1,2,3\}$ is $\sigma_{123}$. In general, $\left|v_{i_{3} i_{4}}\right\rangle_{12}=$ $\sum_{i_{1} i_{2}} \lambda_{i_{1} i_{2} i_{3} i_{4}}\left|i_{1}\right\rangle_{1}\left|i_{2}\right\rangle_{2}$ are linearly independent and they will span the support of $\rho_{12}$.

Hence, any pure state $|\psi\rangle_{1235}$ which agrees with $|\phi\rangle_{1234}$ in subsystem $\{1,2\}$ can be expanded as follows:

$$
\begin{aligned}
|\psi\rangle_{1235} & \\
= & \sum_{i_{3}, i_{4}}\left|v_{i_{3} i_{4}}\right\rangle_{12}\left|E_{i_{3} i_{4}}\right\rangle_{35} \\
& =\sum_{i_{3}, i_{4}} \sum_{i_{1}, i_{2}} \lambda_{i_{1} i_{2} i_{3} i_{4}}\left|i_{1}\right\rangle_{1}\left|i_{2}\right\rangle_{2} \sum_{i_{3}^{\prime}}\left|i_{3}^{\prime}\right\rangle_{3}\left|e_{i_{3} i_{4}, i_{3}^{\prime}}\right\rangle_{5} \\
& =\sum_{\substack{i_{1}, i_{2}, i_{3}, i_{4}, j_{3}}} \lambda_{i_{1} i_{2} i_{3} i_{4}}\left|i_{1}\right\rangle_{1}\left|i_{2}\right\rangle_{2}\left|j_{3}\right\rangle_{3}\left|e_{i_{3} i_{4}, j_{3}}\right\rangle_{5} .
\end{aligned}
$$

Recall that $|\psi\rangle_{1235}$ and $|\phi\rangle_{1234}$ agree in subsystem $\{1,3\}$, we will have

$$
\operatorname{tr}_{\{2,5\}}\left(|\psi\rangle\left\langle\left.\psi\right|_{1235}\right)=\operatorname{tr}_{\{2,4\}}\left(|\phi\rangle\left\langle\left.\phi\right|_{1234}\right) .\right.\right.
$$

Substituting Eqs. (B1) and (B2) into Eq. (B3), and comparing each matrix element, results in the following equalities (for all $i_{1}, i_{1}^{\prime}, j_{3}, j_{3}^{\prime}$ ):

$$
\sum_{\substack{i_{2}, i_{3}, i_{4}, i_{3}^{\prime}, i_{4}^{\prime}}} \lambda_{i_{1} i_{2} i_{3} i_{4}} \lambda_{i_{1}^{\prime} i_{2} i_{3}^{\prime} i_{4}^{\prime}}^{*}\left\langle e_{i_{3}^{\prime} i_{4}^{\prime}, j_{3}^{\prime}} \mid e_{i_{3} i_{4}, j_{3}}\right\rangle=\sum_{i_{2}, j_{4}} \lambda_{i_{1} i_{2} j_{3} j_{4}} \lambda_{i_{1}^{\prime} i_{2} j_{3}^{\prime} j_{4}}^{*} .
$$

Similarly, following from the fact that $|\psi\rangle_{1235}$ and $|\phi\rangle_{1234}$ agree in subsystem $\{1,2\}$, we have the equations

$$
\sum_{\substack{i_{3}, i_{4}, j_{3}, i_{3}^{\prime}, i_{4}^{\prime}}} \lambda_{i_{1} i_{2} i_{3} i_{4}} \lambda_{i_{1}^{\prime} i_{2}^{\prime} i_{3}^{\prime} i_{4}^{\prime}}^{*}\left\langle e_{i_{3}^{\prime} i_{4}^{\prime}, j_{3}} \mid e_{i_{3} i_{4}, j_{3}}\right\rangle=\sum_{i_{3}, i_{4}} \lambda_{i_{1} i_{2} i_{3} i_{4}} \lambda_{i_{1}^{\prime} i_{2}^{\prime} i_{3} i_{4}}^{*}
$$

for any $i_{1}, i_{2}, i_{1}^{\prime}, i_{2}^{\prime}$.

Let us denote $x_{p_{3}, p_{4}, q_{3}, p_{3}^{\prime}, p_{4}^{\prime}, q_{3}^{\prime}}=\left\langle e_{p_{3} p_{4}, q_{3}} \mid e_{p_{3}^{\prime} p_{4}^{\prime}, q_{3}^{\prime}}\right\rangle$ for any $p_{3}, p_{4}, q_{3}, p_{3}^{\prime}, p_{4}^{\prime}, q_{3}^{\prime}$. If there is only one solution $\left\{x_{p_{3}, p_{4}, q_{3}, p_{3}^{\prime}, p_{4}^{\prime}, q_{3}^{\prime}}\right\}$ that satisfies the above two linear systems, then

$$
\begin{aligned}
\sigma_{123}= & \operatorname{tr}_{\{5\}}|\psi\rangle\left\langle\left.\psi\right|_{1235}\right. \\
= & \sum_{\substack{i_{1}, i_{2}, i_{3}, i_{4}, j_{3}, i_{1}^{\prime}, i_{2}^{\prime}, i_{3}^{\prime}, i_{4}^{\prime}, j_{3}^{\prime}}} \lambda_{i_{1} i_{2} i_{3} i_{4}} \lambda_{i_{1}^{\prime} i_{2}^{\prime} i_{3}^{\prime} i_{4}^{\prime}}^{*}\left\langle e_{i_{3}^{\prime} i_{4}^{\prime}, j_{3}^{\prime}} \mid e_{i_{3} i_{4}, j_{3}}\right\rangle \\
& \times\left|i_{1}\right\rangle\left\langle\left. i_{1}^{\prime}\right|_{1} \otimes \mid i_{2}\right\rangle\left\langle\left. i_{2}^{\prime}\right|_{2} \otimes \mid j_{3}\right\rangle\left\langle\left. j_{3}^{\prime}\right|_{3}\right.
\end{aligned}
$$

is completely determined.

There are $d_{1}^{2} d_{3}^{2}+d_{1}^{2} d_{2}^{2}$ linear equations and $d_{3}^{4} d_{4}^{2}$ variables. Under our assumption, we have $d_{3}^{4} d_{4}^{2} \leqslant d_{3}^{4}(\operatorname{rank} \rho)^{2} \leqslant$ $d_{1}^{2} d_{3}^{2}+d_{1}^{2} d_{2}^{2}$. Similar to the proof of Theorem 3 , the coefficient matrix generically has full rank which implies that there is at most one solution. Thus, a generic low-rank density operator $\rho_{123}$ is UDA by its 2 -RDMs of particle pairs $\{1,2\}$ and $\{1,3\}$. 


\section{APPENDIX C: PROOF OF THEOREM 5}

We begin by presenting without proof a result that was proved as Theorem 1(i) in Ref. [22]. Before we state the result, recall from Sec. IV that $W_{2}(A)$ is the numerical range of $A_{1}+$ $i A_{2}$ and is thus convex by the Hausdorff-Toeplitz theorem [20,21]. It therefore makes sense to talk about extreme points of $W_{2}(A)$ in this case.

Proposition 1. Let $\vec{x} \in W_{2}(A)$. Then $\vec{x}$ is an extreme point of $W_{2}(A)$ if and only if

$$
M_{x}:=\{\lambda|\psi\rangle: \lambda \in \mathbb{C}, \mathbf{A}(|\psi\rangle)=\vec{x}\}
$$

is a linear subspace of $\mathcal{H}_{d}$.

We are now in a position to prove Theorem 5.

Proof of Theorem 5. Suppose that $|\psi\rangle$ is UDP and define $\vec{x}:=\left(\left\langle\psi\left|A_{1}\right| \psi\right\rangle,\left\langle\psi\left|A_{2}\right| \psi\right\rangle\right)$. Then $M_{x}=\{\lambda|\psi\rangle: \lambda \in \mathbb{C}\}$, which is linear, so $\vec{x}$ is an extreme point of $W_{2}(A)$ by Proposition 1 . Because $W_{2}(A)=C_{2}(A)$ in this case by convexity, $\vec{x}$ is also an extreme point of $C_{2}(A)$. Suppose now that there exists a mixed state $\rho=\sum_{i} p_{i}\left|\psi_{i}\right\rangle\left\langle\psi_{i}\right|$ with $\vec{x}=\left(\operatorname{Tr}\left(A_{1} \rho\right), \operatorname{Tr}\left(A_{2} \rho\right)\right)$. Then, $\vec{x}=\sum_{i} p_{i}\left(\left\langle\psi_{i}\left|A_{1}\right| \psi_{i}\right\rangle,\left\langle\psi_{i}\left|A_{2}\right| \psi_{i}\right\rangle\right)$. Since $\vec{x}$ is extreme in $C_{2}(A)$, it follows that $\vec{x}=\left(\left\langle\psi_{i}\left|A_{1}\right| \psi_{i}\right\rangle,\left\langle\psi_{i}\left|A_{2}\right| \psi_{i}\right\rangle\right)$ for all $i$, which contradicts the fact that $|\psi\rangle$ is UDP unless each $\left|\psi_{i}\right\rangle$ is the same up to global phase (i.e., $\rho=|\psi\rangle\langle\psi|$ ).

Based on the proof of Theorem 5, we might expect that UDP implies UDA for all pure states whenever $W_{m}(\mathbf{A})$ is convex. However, the example provided in Sec. IV showed this not to be the case. We now expand upon the reason for this apparent discrepancy, which lies buried in the proof of Proposition 1.

In the case when $W_{m}(\mathbf{A})$ is convex, the "only if" implication of Proposition 1 still holds for arbitrary $m$. However, the proof of the "if" implication relies on the fact that if $\vec{x}:=\mathbf{A}(|\phi\rangle)$ and $\vec{y}:=\mathbf{A}(|\psi\rangle)$, then for any $s \in(0,1)$ we can find $\alpha, \beta \in \mathbb{C}$ such that $s \vec{x}+(1-s) \vec{y}=\mathbf{A}(\alpha|\phi\rangle+\beta|\psi\rangle)$. In other words, the proof of the proposition uses the fact that $W_{m}(\mathbf{A})$ is not only convex, but that convex combinations are in a sense well behaved between the input and output of $\mathbf{A}(\cdots)$. For convenience, we refer to this property as strong convexity for the remainder of this section.

The standard proofs of the Hausdorff-Toeplitz theorem show that strong convexity, not just convexity itself, always holds when $m=2$. To see how strong convexity can fail when $m>2$ even when convexity holds, we again return to the example of Sec. IV. In this case, we have $\mathbf{A}(|0\rangle)=(0,0,1)$ and $\mathbf{A}(|1\rangle)=(0,0,-1)$. However, even though $W_{3}(A)$ is convex and thus there exists a pure state $|\psi\rangle$ with $\mathbf{A}(|\psi\rangle)=(0,0,0)$, the only such pure state is $|\psi\rangle:=|2\rangle$, which is not contained in the span of $|0\rangle$ and $|1\rangle$.

We might hope that strong convexity, rather than convexity itself, provides the natural generalization of Theorem 5. That is, we might hope that if $W_{m}(\mathbf{A})$ is strongly convex, then UDP implies UDA for all pure states. It turns out that this is a true but vacuous statement: if $W_{m}(\mathbf{A})$ is strongly convex then it must be the case that $m \leqslant 2$, so Theorem 5 itself applies directly. This fact seems to be implicit in many papers on the joint numerical range, but we prove it here for completeness.

Before stating the result, we briefly note that we can assume without loss of generality that $\mathbf{A}$ contains $I$ and is linearly independent, as adding the identity to $\mathbf{A}$ has no effect on convexity, UDA, or UDP, and furthermore these properties only depend on the span of the observables in $\mathbf{A}$.

Proposition 2. Let $\mathbf{A}=\left(I, A_{1}, \ldots, A_{m}\right)$ be a linearly independent set. Then $W_{m+1}(\mathbf{A})$ is strongly convex if and only if $m \leqslant 2$.

Proof. The "if" direction, as already mentioned, follows from any of the usual proofs of the Hausdorff-Toeplitz theorem.

For the "only if" direction, suppose that that $W_{m+1}(\mathbf{A})$ is strongly convex and assume (in order to get a contradiction) that $m \geqslant 3$. By ([24], Theorem 4.1), there exists $X \in M_{d, 2}$ with $X^{*} X=I$ such that $X^{*} \mathbf{A} X:=\left\{I, X^{*} A_{1} X, \ldots, X^{*} A_{m} X\right\}$ spans all of $M_{2}$. By letting $|\phi\rangle$ and $|\psi\rangle$ be the column vectors of $X$, we see that strong convexity of $W_{m+1}(\mathbf{A})$ immediately implies convexity of $W_{m+1}\left(X^{*} \mathbf{A} X\right)$. Since convexity of $W_{m+1}\left(X^{*} \mathbf{A} X\right)$ depends only on the span of $X^{*} \mathbf{A} X$, it follows that $W_{4}(\mathbf{B})$ is also convex, where $\mathbf{B}:=\left\{\lambda_{0}, \lambda_{1}, \lambda_{2}, \lambda_{3}\right\}$ is the Pauli basis given by Eq. (8). However, it is easily verified that $W_{4}(\mathbf{B})$ is the Bloch sphere embedded in four-dimensional space and hence is not convex, which gives the desired contradiction.

It thus seems that numerical range and convexity arguments are not able to tell us anything nontrivial about the UDP and UDA problem beyond the $m=2$ case of Theorem 5 .

\section{APPENDIX D: SYMMETRIES AND UDP AND UDA: PROOF OF THEOREM 6}

We will see that if there is a compact group of symmetries whose fixed-point set is the linear span of observables $\left(A_{1}, \ldots, A_{m}\right)$, then UDP for these observables implies UDA for any pure state. We start with the following result about fixed points of compact groups.

Theorem 9. Let $G$ be a compact group of unitaries on a real or complex finite-dimensional Hilbert space $H$, and let $L$ be the set of fixed points of $G$. Let $\mu$ be Haar measure on $G$, and define $P: H \rightarrow H$ to be the linear map satisfying

$$
\langle P \xi, \eta\rangle=\int_{G}\langle g \xi, \eta\rangle d \mu(g)
$$

for $\xi, \eta \in H$. Then $P$ is the orthogonal projection onto $L$, $P g=g P=P$ for all $g \in G$, and $P$ is in the convex hull of $G$.

Proof. Left and right invariances of Haar measure imply that $P g=g P=P$ for all $g \in G$. The definition of $P$ implies that $L \subset$ im $P$. Now, $g P=P$ for all $g \in G$ implies im $P \subset L$, and hence im $P=L$. Next, im $P=L$ and the definition of $P$ give $P^{2}=P$. To show that $P^{\dagger}=P$ we use the fact that the integrals of $f(g)$ and $f\left(g^{-1}\right)$ are the same for Haar measure, together with the assumption that $G$ is a group of unitaries:

$$
\begin{aligned}
\langle\xi, P \eta\rangle & =\langle P \eta, \xi\rangle^{*}=\int_{G}\langle g \eta, \xi\rangle^{*} d \mu(g) \\
& =\int_{G}\langle\xi, g \eta\rangle d \mu(g)=\int_{G}\left\langle g^{\dagger} \xi, \eta\right\rangle d \mu(g) \\
& =\int_{G}\left\langle g^{-1} \xi, \eta\right\rangle d \mu(g)=\int_{G}\langle g \xi, \eta\rangle d \mu(g) \\
& =\langle P \xi, \eta\rangle .
\end{aligned}
$$

Finally, by the Alaoglu-Birkhoff mean ergodic theorem ([25], Proposition 4.3.4) $P$ is in the strong closure of the convex hull 
of $G$. Since $H$ is finite dimensional, then the space of linear operators on $H$ is also finite dimensional, so the convex hull of the compact set $G$ is compact and hence closed.

Symmetries of $K_{d}$ are given by conjugation by unitaries or by the transpose map or by composition of these two types of symmetries. (An affine automorphism of $K_{d}$ preserves transition probabilities, cf. [26], so this is a consequence of Wigner's theorem [27].)

If we view the space of observables in $M_{d}$ as a real Hilbert space [with the usual inner product $\langle X, Y\rangle=\operatorname{tr}(X Y)$ ], then conjugation by unitaries and the transpose map both preserve this inner product, so are given by unitaries on this Hilbert space.

If $L$ is a (real) linear subspace of observables containing the identity, then $L$ will be the real linear span of $L \cap K_{d}$. Thus, any symmetry of $K_{d}$ will fix $L \cap K_{d}$ if and only if that symmetry when extended to a map on $M_{d}$ fixes $L$. If $G$ is a compact group of symmetries whose fixed-point set is $L \cap$ $K_{d}$, then the corresponding maps on $M_{d}$ will have fixed-point set $L$.

Theorem 10. Let $A$ be a finite set of observables on $H_{d}$ with real linear span $L$. Assume there exists a compact group $G$ of affine automorphisms of $K_{d}$ whose fixed-point set is $L \cap K_{d}$. Then, each pure state which is UDP for measuring $A$ is also UDA.

Proof. As discussed above, we may view $G$ as a compact group of unitaries with fixed-point set $L$. Define $P$ as in Theorem 9. Fix a pure state $\rho$.

Suppose first that $\rho \notin L \cap K_{d}$. Then, there is some $g \in G$ such that $g(\rho) \neq \rho$. Since $P g=P$, both $g(\rho)$ and $\rho$ are pure states with the same image in $L \cap K_{d}$ under the map $P$. Thus, UDP fails for $\rho$ (and hence trivially UDA fails). Now suppose $\rho \in L \cap K_{d}$. Let $\sigma \in K_{d}$ be a preimage of $\rho$ under $P$. Then

$1=\langle P \sigma, \rho\rangle=\left|\int_{G}\langle g \sigma, \rho\rangle d \mu(g)\right| \leqslant \int_{G}\|g \sigma\|\|\rho\| d \mu(g) \leqslant 1$,

and equality can hold only if $g \sigma=\rho$ for all $g$, i.e., if and only if $\sigma \in L$. Then, $\sigma=P \sigma=\rho$, so for such $\rho$ both UDP and UDA hold.

Corollary 1. For $d=2$, for all pure states and all sets $\mathbf{A}$ of observables, UDP implies UDA.

Proof. Let $A_{1}=I, A_{2}, \ldots, A_{m}$ be observables in $M_{2}$ and let $L=S(A)$ be their real linear span. We will show that there is a finite group of affine automorphisms $G$ of the state space $K_{d}$ of $M_{2}$ with fixed-point set $L \cap K_{d}$. There are three cases, depending on the dimension of the fixed-point set. The fixed-point set in the Bloch sphere will be the central point, a diameter of the Bloch sphere, or the intersection of a plane (through the center) with the Bloch sphere. In each case, reflection of the Bloch sphere in the fixed-point set generates an order-2 group of affine automorphisms with fixed-point set $L \cap K_{d}$. Now, the corollary follows from Theorem 10 .

Corollary 2. Let $\mathbf{A}=A_{1}, \ldots, A_{p}$ be observables in $M_{d}$. If the (complex) linear span of $\mathbf{A}$ is a ${ }^{*}$-subalgebra $\mathcal{A}$ of $M_{d}$, then $\mathrm{UDP}=\mathrm{UDA}$ for pure states measured by these observables.

Proof. UDP and UDA for a set of observables are not affected if we include the identity among those observables, so hereafter we assume that $I_{d} \in \mathbf{A}$. Note that $\mathcal{A}$ is the linear span of the unitaries in $\mathcal{A}$. Furthermore, $\mathcal{A}$ is a von Neumann algebra containing the identity $I_{d}$, so by the bicommutant theorem ([28], Theorem 2.77) $\left(\mathcal{A}^{\prime}\right)^{\prime}=\mathcal{A}$, where for $X \subset M_{d}, X^{\prime}$ denotes the algebra of matrices that commute with all matrices in $X$. Combining these two statements shows that $\mathcal{A}$ is the set of matrices that commute with all unitaries in $\mathcal{A}^{\prime}$, and thus is the set of fixed points of $G=\left\{\operatorname{Ad}_{U} \mid \mathrm{U}\right.$ is a unitary in $\left.\mathcal{A}^{\prime}\right\}$. It follows that $L=\mathcal{A}_{s a}$ (the Hermitian matrices in $\mathcal{A}$ ) is the set of observables fixed by the compact group $G$. The corollary follows from Theorem 10.

Example 1. Let $\mathbf{A}=\left\{E_{11}, \ldots, E_{d d}\right\}$. Then the complex linear span of $\mathbf{A}$ consists of the diagonal matrices. From Corollary 2 it follows that for each pure state on $M_{d}$, UDP for A implies UDA.

We can generalize the last example by taking the *-algebra consisting of diagonal observables with the restriction that certain diagonal entries coincide. For example, if $d=7$ we can look at diagonal matrices of the form $\operatorname{diag}(a, a, b, b, b, c, d)$ whose linear span will be four dimensional. The space of Hermitian members of this algebra is four dimensional. If we choose four observables including the identity spanning this space, then UDA = UDP for all pure states when measuring these observables. (We could drop the identity from this list if we wish.) In this way for any $d$ we can find a set of $k$ observables for any $k \leqslant d$ for which $\mathrm{UDA}=\mathrm{UDP}$.

We can also find many larger sets of observables for which UDA $=$ UDP. For example, for any $d$ we can consider the $*_{-}$ algebra of all block-diagonal matrices with $k$ blocks that are of size $d_{i} \times d_{i}$ for $1 \leqslant k \leqslant p$, where $d_{1}+d_{2}+\cdots d_{p}=d$. The subspace of Hermitian matrices in this algebra has dimension $\sum_{i} d_{i}^{2}$, so any such dimension is realizable as the number of observables in a set of observables for which UDA = UDP holds for all pure states.
[1] T. Heinosaari, L. Mazzarella, and M. M. Wolf, Commun. Math. Phys. 318, 355 (2013).

[2] S. Weigert, Phys. Rev. A 45, 7688 (1992).

[3] J.-P. Amiet and S. Weigert, J. Phys. A: Math. Gen. 32, 2777 (1999).

[4] J. Finkelstein, Phys. Rev. A 70, 052107 (2004).

[5] S. T. Flammia, A. Silberfarb, and C. M. Caves, Found. Phys. 35, 1985 (2005).

[6] D. Gross, Y.-K. Liu, S. T. Flammia, S. Becker, and J. Eisert, Phys. Rev. Lett. 105, 150401 (2010).
[7] M. Cramer, M. B. Plenio, S. T. Flammia, R. Somma, D. Gross, S. D. Bartlett, O. Landon-Cardinal, D. Poulin, and Y.-K. Liu, Nat. Commun. 1, 149 (2010).

[8] W.-T. Liu, T. Zhang, J.-Y. Liu, P.-X. Chen, and J.-M. Yuan, Phys. Rev. Lett. 108, 170403 (2012).

[9] J. Chen, Z. Ji, B. Zeng, and D. L. Zhou, Phys. Rev. A 86, 022339 (2012).

[10] J. Chen, Z. Ji, M. B. Ruskai, B. Zeng, and D.-L. Zhou, J. Math. Phys. 53, 072203 (2012).

[11] N. Linden, S. Popescu, and W. K. Wootters, Phys. Rev. Lett. 89, 207901 (2002). 
[12] L. Diósi, Phys. Rev. A 70, 010302 (2004).

[13] N. S. Jones and N. Linden, Phys. Rev. A 71, 012324 (2005).

[14] P. Parashar and S. Rana, Phys. Rev. A 80, 012319 (2009).

[15] J. Chen, Z. Ji, Z. Wei, and B. Zeng, Phys. Rev. A 85, 040303 (2012).

[16] S. N. Walck and D. W. Lyons, Phys. Rev. Lett. 100, 050501 (2008).

[17] N. Linden and W. K. Wootters, Phys. Rev. Lett. 89, 277906 (2002).

[18] T. Cubitt, A. Montanaro, and A. Winter, J. Math. Phys. 49, 022107 (2008).

[19] J. Chen, Z. Ji, A. Klyachko, D. W. Kribs, and B. Zeng, J. Math. Phys. 53, 022202 (2012).

[20] O. Toeplitz, Math. Z. 2, 187 (1918).
[21] F. Hausdorff, Math. Z. 3, 314 (1919).

[22] M. R. Embry, Pacific J. Math. 32, 647 (1970).

[23] R. A. Horn and C. R. Johnson, Matrix Analysis (Cambridge University Press, Cambridge, 1990).

[24] C.-K. Li and Y.-T. Poon, SIAM J. Matrix Analysis Appl. 21, 668 (1999).

[25] O. Bratteli and D. Robinson, Operator Algebras and Quantum Statistical Mechanics I, 2nd ed. (Springer, Heidelberg, 2003).

[26] F. Shultz, Commun. Math. Phys. 82, 497 (1982).

[27] E. P. Wigner, Group Theory and its Application to the Quantum Mechanics of Atomic Spectra (Academic, New York, 1959), translated from the original by J. J. Griffin.

[28] E. Alfsen and F. Shultz, State Spaces of Operator Algebras: Basic Theory, Orientations and $C^{*}$-products (Birkhäuser, Boston, 2001). 